\title{
Decreased Leptin Is Associated with Alterations in Thyroid-Stimulating Hormone Levels after Roux-en-Y Gastric Bypass Surgery in Obese Euthyroid Patients with Type 2 Diabetes
}

\author{
Haoyong $\mathrm{Yu}^{\mathrm{a}, \mathrm{b}}$ Qing $\mathrm{Li}^{\mathrm{a}, \mathrm{b}}$ Mingliang Zhang ${ }^{\mathrm{a}, \mathrm{b}}$ Fangyuan Liu ${ }^{\mathrm{a}, \mathrm{b}}$ \\ Jiemin Pan ${ }^{a, b}$ Yinfang Tu ${ }^{a, b}$ Junxi Lu ${ }^{a, b}$ Pin Zhang ${ }^{c}$ Junfeng Han ${ }^{a, b}$ \\ Weiping Jia ${ }^{a, b}$ Yuqian Bao ${ }^{a, b}$ \\ aDepartment of Endocrinology and Metabolism, Shanghai Sixth People's Hospital, \\ affiliated to Shanghai Jiao Tong University, Shanghai, China; ${ }^{b}$ Shanghai Diabetes Institute, \\ Shanghai Clinical Center of Diabetes, Shanghai Key Laboratory of Diabetes Mellitus, \\ Shanghai Key Clinical Center for Metabolic Diseases, Shanghai, China; 'Department of \\ General Surgery, Shanghai Sixth People's Hospital, affiliated to Shanghai Jiao Tong \\ University, Shanghai, China
}

Keywords

Bariatric surgery $\cdot$ Thyroid-stimulating hormone $\cdot$ Leptin

\begin{abstract}
Background: Leptin has been shown to stimulate the hypothalamus-pituitary-thyroid (HPT) axis in vivo and vitro. Its role in thyroid axis regulation after weight loss induced by bariatric surgery is still unknown. The aim of this study was to evaluate the influence of leptin on weight loss and thyroid function variation induced by Roux-en-Y gastric bypass (RYGB) surgery in euthyroid individuals with obesity and type 2 diabetes mellitus (T2DM). Methods: 65 Chinese individuals with obesity and T2DM who underwent RYGB, and 27 healthy volunteers were enrolled in this retrospective study. Participants were evaluated for changes in anthropometric parameters, metabolic indexes, thyroid function, and leptin levels before and 12 months after surgery. Results: After RYGB, all of these patients experienced significant weight reduction and improved glucose control. Metabolic parameters were significantly ameliorated after surgery compared with baseline. Thyroid hormones including free triiodothyronine $\left(\mathrm{FT}_{3}\right)$, free thyroxine $\left(\mathrm{FT}_{4}\right)$, and thyroid-stimulating hormone (TSH) declined in parallel. Median (IQR) plasma leptin levels decreased from $33.7 \mathrm{ng} / \mathrm{mL}$ (17.9-63.1) to $10.3 \mathrm{ng} / \mathrm{mL}$ (4.0-18.5). Pearson
\end{abstract}

H.Y. and Q.L. contributed equally to this work. 
correlation analysis showed that TSH was significantly positively correlated with body mass index, $C$-reactive protein (CRP), and leptin. Multiple stepwise linear regression indicated that leptin and CRP were independent factors affecting TSH. The $\beta$ coefficients were 0.38 ( $p=$ $0.001)$ and $0.32(p=0.004)$, respectively. There was a significant positive correlation between $\Delta \mathrm{TSH}$ and $\Delta$ leptin $(r=0.33, p=0.01)$. Conclusion: Decreased or normalized TSH levels after weight loss induced by RYGB might be mediated by the decline in leptin. There could be cross talk between adipose tissue and the HPT axis.

(C) 2019 The Author(s)

Published by S. Karger AG, Basel

\section{Introduction}

Obesity is one of the most common diseases in many countries, affecting $>600$ million adults worldwide [1]. Sustained imbalance between energy intake and energy output plays a pivotal role in the progress of obesity. As a systemic disease, obesity can cause a series of comorbidities and weaken multiple organ functions. Various abnormalities of the endocrine system, such as the hyperactivity of the hypothalamic-pituitary-adrenal axis, impaired growth hormone response to established stimuli, and thyroid dysfunction have been observed in patients with obesity [2]. Within the normal range, serum TSH is significantly and positively correlated with body mass index (BMI). In addition, weight loss in these individuals was associated with changes in serum thyroid-stimulating hormone (TSH) and thyroid hormone levels [2-4]. Meanwhile, type 2 diabetes mellitus (T2DM) is the most common chronic endocrine disease, characterized by hyperglycemia resulting from impaired insulin secretion and/or insulin resistance [5]. Recently, increased interest has been focused on the relationship between thyroid function and T2DM. Decreased free triiodothyronine $\left(\mathrm{FT}_{3}\right)$ and free thyroxine $\left(\mathrm{FT}_{4}\right)$ levels were independently related to the prevalence of T2DM among the adult population. A significant negative relationship between TSH and T2DM was observed in male but not in female individuals [6].

Bariatric surgery is currently one of the most effective treatments to achieve long-term weight reduction. Roux-en-Y gastric bypass (RYGB) surgery, a commonly performed operation, can bring about long-term weight loss and excellent remission of associated comorbidities and metabolic disorders [7, 8]. Inconsistent thyroid hormone level alterations in patients with obesity after bariatric surgery were observed in several studies mainly due to different study populations, sample sizes, and surgical procedures $[9,10]$, emphasizing the need for further research.

The relationship between adipose tissue and thyroid function remains uncertain. The complex interaction between thyroid hormones and adipose tissue involves TSH and thyroid hormones which further participate in adipocyte differentiation and lipolysis regulation [11], while various adipocyte cytokines interact with the hypothalamus-pituitary-thyroid (HPT) axis. Leptin, secreted by adipose tissue, is one component of a regulatory loop linking fat mass to food intake and energy expenditure [12]. Furthermore, leptin plays an important role in the regulation of the HPT axis by controlling thyrotropin-releasing hormone gene expression in the paraventricular nucleus. TSH in turn stimulates leptin secretion by adipose tissue [13]. The mutual relationship between leptin and the HPT axis in humans has been an area of great scientific interest.

It is not clear whether leptin participates in the process of weight loss and thyroid hormone level alterations after bariatric surgery, especially in the context of T2DM. Therefore, our aim was to evaluate the effect of leptin on weight loss and thyroid function variation induced by RYGB in euthyroid individuals with obesity and T2DM. 
Yu et al.: Leptin and TSH Alterations after RYGB

\section{Methods}

\section{Study Population}

This is a retrospective study; 65 euthyroid individuals diagnosed with obesity and T2DM and 27 healthy controls matched for gender and age were enrolled in this study. Age, height, weight, BMI, medical history, and current medications were recorded before and after surgery. Levels of glucose, hemoglobin $\mathrm{A}_{1 \mathrm{c}}\left(\mathrm{HbA}_{1 \mathrm{c}}\right)$, C-peptide, thyroid hormones, and other biochemical parameters were detected at baseline and 12 months after the operation. Exclusion criteria included (1) abnormal levels of thyroid hormones, (2) a history of thyroid disease, including hyper- and hypothyroidism, (3) previous diagnosis of pituitary-related disorders, and (4) drug therapy affecting serum levels of thyroid hormones in the past 3 months.

According to the World Medical Association's recommendations, this study was approved by the Sixth People's Hospital Ethics Committee (Shanghai Jiao Tong University). All participants gave written informed consent before the study.

The retrospective study identifier in clinicaltrials.gov is NCT02217943.

\section{Surgical Technique}

All surgical procedures, using a previously reported standardized technique, were performed laparoscopically by a single surgeon [14]. A 25-mL gastric pouch was separated from the distal remnant. The lengths of the biliopancreatic and alimentary limbs were $100-120 \mathrm{~cm}$.

\section{Laboratory Measurements}

Blood samples were collected from all enrolled subjects after an overnight fast in order to assess biochemical and hormonal parameters. Serum lipid profiles were determined by standard enzymatic methods using a Hitachi 747 analyzer (Castle Hill, NSW, Australia). Plasma glucose concentrations were assessed using the glucose oxidase method on a Hitachi 747 analyzer. High-performance liquid chromatography (Bio-Rad Laboratories, Hercules, CA, USA) was used to detect $\mathrm{HbA}_{1 \mathrm{c}}$. Serum C-peptide was measured by radioimmunoassay using specific detection kits according to the manufacturer's instructions (Beijing North Institute of Biological Technology, Beijing, China). Serum $\mathrm{FT}_{3}, \mathrm{FT}_{4}$, and $\mathrm{TSH}$ were all detected applying the electrochemiluminescence immunoassay. The normal ranges of serum $\mathrm{FT}_{3}, \mathrm{FT}_{4}$, and $\mathrm{TSH}$ levels are 3.1-6.8 pmol/L, 12.0-22.0 pmol/L, and 0.27-4.20 mIU/L, respectively. The intraand interassay coefficients of the 2 indexes were $<10 \%$. Serum C-reactive protein (CRP) level was measured by particle-enhanced immunonephelometry (Dade Behring Inc., Newark, NJ, USA). The lower detection limit of the assay was $0.15 \mathrm{mg} / \mathrm{L}$. Human leptin was measured by quantitative sandwich ELISA (BioVendor, Brno, Czech Republic; lot No. E14-025), in accordance with the manufacturer's instructions. Intra- and interassay variability of the kit was 4.2 and $6.7 \%$, respectively.

\section{Calculations}

BMI was computed as weight $(\mathrm{kg})$ divided by height $\left(\mathrm{m}^{2}\right)$. HOMA-IR (homeostatic model assessment for insulin resistance) was calculated as fasting insulin $(\mathrm{mIU} / \mathrm{L}) \times$ fasting glucose $(\mathrm{mM}) / 22.5$. The changes in TSH $(\Delta \mathrm{TSH}), \mathrm{BMI}(\Delta \mathrm{BMI}), \mathrm{HbA}_{1 \mathrm{c}}\left(\Delta \mathrm{HbA}_{1 \mathrm{c}}\right), \mathrm{CRP}(\Delta \mathrm{CRP})$, and leptin $(\Delta$ leptin) were deemed as the values 12 months after surgery minus the values at baseline.

\section{Statistical Analysis}

All continuous variables were tested for normal distribution using the KolmogorovSmirnov normality test. Clinical characteristics and metabolic variables were presented as 
Yu et al.: Leptin and TSH Alterations after RYGB

Table 1. Clinical and biochemical parameters of obese patients with T2DM before and 1 year after RYGB and controls

\begin{tabular}{|c|c|c|c|}
\hline \multirow[t]{2}{*}{ Variables } & \multicolumn{2}{|l|}{ Patients } & \multirow[t]{2}{*}{ Controls } \\
\hline & baseline & 12 months after surgery & \\
\hline Subjects, $n$ (females/males) & $65(34 / 31)$ & - & $27(17 / 10)$ \\
\hline Age, years & $39.9 \pm 10.3$ & - & $40.3 \pm 9.3$ \\
\hline Diabetes duration, years & $4.0(1.0-6.5)$ & - & - \\
\hline Body mass index & $32.5 \pm 4.6$ & $23.1 \pm 3.2^{* *}$ & $22.8 \pm 2.2^{* *}$ \\
\hline \multicolumn{4}{|l|}{ Blood pressure, $\mathrm{mm} \mathrm{Hg}$} \\
\hline Systolic & $130.6 \pm 15.1$ & $118.8 \pm 12.9^{* *}$ & $111.4 \pm 12.5^{* *, \# \#}$ \\
\hline Diastolic & $82.9 \pm 10.2$ & $75.7 \pm 9.3^{* *}$ & $73.2 \pm 9.5^{* *, \# \#}$ \\
\hline Alanine transaminase, $\mathrm{U} / \mathrm{L}$ & $35.0(19.0-47.0)$ & $19.0(14.0-28.0)^{* *}$ & $13.5(10.0-23.0)^{* *, \#}$ \\
\hline Aspartate transaminase, U/L & $21.0(16.5-31.5)$ & $19.0(16.0-24.0)^{* *}$ & $17.5(14.8-20.0)^{* *}$ \\
\hline BUN, mmol/L & $4.6 \pm 1.1$ & $4.7 \pm 1.3$ & $4.8 \pm 1.1$ \\
\hline Total cholesterol, mmol/L & $5.1 \pm 1.2$ & $4.1 \pm 0.7^{* *}$ & $4.2 \pm 0.7^{* *}$ \\
\hline Triglycerides, mmol/L & $1.9(1.4-2.9)$ & $0.9(0.7-1.2)^{* *}$ & $0.9(0.7-1.3)^{* *}$ \\
\hline HDL-cholesterol, mmol/L & $1.0 \pm 0.2$ & $1.3 \pm 0.3^{* *}$ & $1.4 \pm 0.3^{* *, \# \#}$ \\
\hline LDL-cholesterol, mmol/L & $3.0 \pm 1.0$ & $2.3 \pm 0.6^{* *}$ & $2.6 \pm 0.6^{* *}$ \\
\hline \multicolumn{4}{|l|}{ Plasma glucose, $\mathrm{mmol} / \mathrm{L}$} \\
\hline Fasting & $8.2 \pm 2.7$ & $5.3 \pm 1.0 * *$ & $5.3 \pm 0.4^{* *}$ \\
\hline $2 \mathrm{~h}$ after a meal & $12.1 \pm 4.0$ & $6.2 \pm 2.7^{* *}$ & $5.7 \pm 1.3^{* *}$ \\
\hline $\mathrm{HbA}_{1 \mathrm{c}}, \%$ & $7.6 \pm 1.7$ & $5.6 \pm 0.6^{* *}$ & $5.5 \pm 0.2^{* *}$ \\
\hline HOMA-IR & $6.0(3.7-10.5)$ & $1.4(0.9-2.1)^{* *}$ & $1.6(1.1-2.2)^{* *}$ \\
\hline \multicolumn{4}{|l|}{ C-peptide, ng/mL } \\
\hline Baseline & $2.8(2.4-3.7)$ & $1.8(1.5-2.2)^{* *}$ & $1.8(1.3-2.3)^{* *}$ \\
\hline $2 \mathrm{~h}$ & $6.5(4.2-10.2)$ & $5.1(3.1-7.7)^{* *}$ & $5.5(3.8-7.0)^{* *}$ \\
\hline C-reactive protein, mg/L & $2.5(1.4-4.3)$ & $0.3(0.2-0.6)^{* *}$ & $0.5(0.2-1.0)^{* *}$ \\
\hline $\mathrm{TSH}, \mathrm{mIU} / \mathrm{L}$ & $2.2(1.3-3.0)$ & $1.6(1.1-2.5)^{*}$ & $1.8(1.2-2.4) *$ \\
\hline $\mathrm{FT}_{3}, \mathrm{pmol} / \mathrm{L}$ & $4.8 \pm 0.6$ & $4.3 \pm 0.6^{* *}$ & $4.4 \pm 0.5$ \\
\hline $\mathrm{FT}_{4}, \mathrm{pmol} / \mathrm{L}$ & $16.2 \pm 2.2$ & $15.5 \pm 2.3^{*}$ & $15.6 \pm 1.9$ \\
\hline Leptin, ng/mL & $33.7(17.9-63.1)$ & $10.3(4.0-18.5)^{* *}$ & $10.1(6.8-18.2)^{* *}$ \\
\hline
\end{tabular}

Normally distributed data are expressed as means $\pm \mathrm{SD}$, nonnormally distributed data are presented as medians (IQR). ${ }^{*} p<0.05,{ }^{* *} p<0.01$, vs. baseline ${ }^{\#} p<0.05,{ }^{\# \#} p<0.0$, vs. 12 months after surgery.

means \pm SD or medians (IQR). The paired-sample or Wilcoxon signed-rank test was applied to compare continuous data at baseline and 12 months after surgery. Clinical characteristics between the obesity with T2DM group and the healthy control group were compared by Student's $t$ test or Mann-Whitney U test. Pearson correlation analysis was used to determine correlations between multiple clinical parameters and TSH. This method was also conducted to assess the mathematical relationship between $\Delta \mathrm{TSH}$ and age, $\Delta$ leptin, $\Delta \mathrm{HbA}_{1 c}$, and $\Delta \mathrm{CRP}$, respectively. Multiple stepwise regression analysis was used to evaluate the independent parameters concerned with TSH. Variables related to TSH with $p<0.2$ confirmed by Pearson correlation were entered into the multiple stepwise regression model. Statistical significance in this study was defined as $p<0.05$ (two-tailed tests). Statistical analyses were performed using SPSS 17.0 (SPSS, Chicago, IL, USA).

\section{Results}

Clinical and biochemical characteristics of participants in the bariatric surgery follow-up study and age- and gender-matched controls are summarized in Table 1. Systolic blood pressure, diastolic blood pressure, serum glucose level, $\mathrm{HbA}_{1 \mathrm{c}}$, C-peptide, CRP, BMI, HOMA-IR, 
Yu et al.: Leptin and TSH Alterations after RYGB

Table 2. Multiple stepwise regression analysis of TSH and variables

\begin{tabular}{lllll}
\hline Variable & B & SE & $\beta$ coefficient & $p$ \\
\hline Leptin & 0.02 & 0.005 & 0.414 & 0.001 \\
C-reactive protein & 0.13 & 0.048 & 0.283 & 0.007
\end{tabular}

Adjusted for body mass index, age, and fasting C-peptide.

Model $R^{2}=0.365$, adjusted $R^{2}=0.349$.

and blood lipid profiles were significantly decreased 12 months after RYGB compared to baseline. Meanwhile, thyroid hormones including $\mathrm{FT}_{3}, \mathrm{FT}_{4}$, and $\mathrm{TSH}$ were decreased in parallel after RYGB. Although thyroid hormone levels were within the normal range after RYGB, TSH was mildly elevated in the surgery group at baseline compared with the normal control group. No significant differences in both $\mathrm{FT}_{3}$ and $\mathrm{FT}_{4}$ levels were found between surgery and control groups. The median (IQR) plasma leptin concentrations were significantly decreased 12 months after RYGB: from $33.7 \mathrm{ng} / \mathrm{mL}$ (17.9-63.1) before RYGB to $10.3 \mathrm{ng} / \mathrm{mL}$ (4.0-18.5) $(p<0.01)$. As expected, leptin level was significantly elevated in the surgery group at baseline. However, 12 months after surgery, leptin level declined and was indistinguishable from the control group.

Pearson correlation analysis showed that TSH was significantly and positively correlated with BMI, CRP, and leptin (Fig. 1). Multiple stepwise linear regression indicated that leptin and CRP were independent factors affecting TSH. The $\beta$ coefficients were $0.41(p=0.001)$ and 0.28 ( $p=0.007)$, respectively, after adjusting for age, BMI, and fasting C-peptide (Table 2). Finally, we found that $\Delta$ TSH was positively correlated with $\Delta$ leptin $(r=0.33 ; p=0.01$ ) (Fig. 2). No significant correlation was found between $\Delta$ TSH and age $(r=-0.16 ; p=0.244), \Delta$ BMI $(r=$ $-0.212 ; p=0.111), \Delta \mathrm{CRP}(r=0.081 ; p=0.570)$, and $\Delta \mathrm{HbA}_{1 \mathrm{c}}(r=-0.016 ; p=0.908)$.

\section{Discussion}

In the current study focusing on obese T2DM individuals with normal thyroid function, we demonstrated that elevated levels of thyroid hormones at baseline were closely associated with RYGB-induced weight loss and blood glucose level normalization after surgery for 12 months. The relationship between thyroid hormone alterations and weight change has been investigated in several prospective and observational studies with controversial results. Moulin de Moraes et al. [15] found that $\mathrm{FT}_{4}$ remained steady and TSH decreased significantly in obese subjects 1 year after RYGB surgery. Comparable results were described by Chikunguwo et al. [16] in a 12-month follow-up study which enrolled 86 euthyroid obese patients after RYGB or adjustable gastric banding surgery. On the contrary, MacCuish et al. [17] reported that weight loss after RYGB was accompanied by a significant increase in serum $\mathrm{FT}_{4}$ but no change in TSH in 55 euthyroid individuals with morbid obesity. Vettor et al. [18] observed a significant decline in both $\mathrm{FT}_{3}$ and $\mathrm{FT}_{4}$, while TSH remained unchanged in an 18-month follow-up study which enrolled 10 morbidly obese subjects receiving biliopancreatic diversion surgery. In the present study, thyroid hormone levels including $\mathrm{FT}_{3}, \mathrm{FT}_{4}$, and TSH were all decreased 12 months after RYGB and similar to control levels. A recent systematic review reported that decreases in $\mathrm{TSH}, \mathrm{FT}_{3}$, and $\mathrm{T}_{3}$ were expected following bariatric surgery, as well as nonsignificant changes in $\mathrm{T}_{4}, \mathrm{FT}_{4}$, and $\mathrm{rT}_{3}$ levels [19]. These inconsistent results might be due to the differences in subject populations, sample sizes, and surgical procedures. It is possible that the obese individuals with normal thyroid function involved in our study 
A

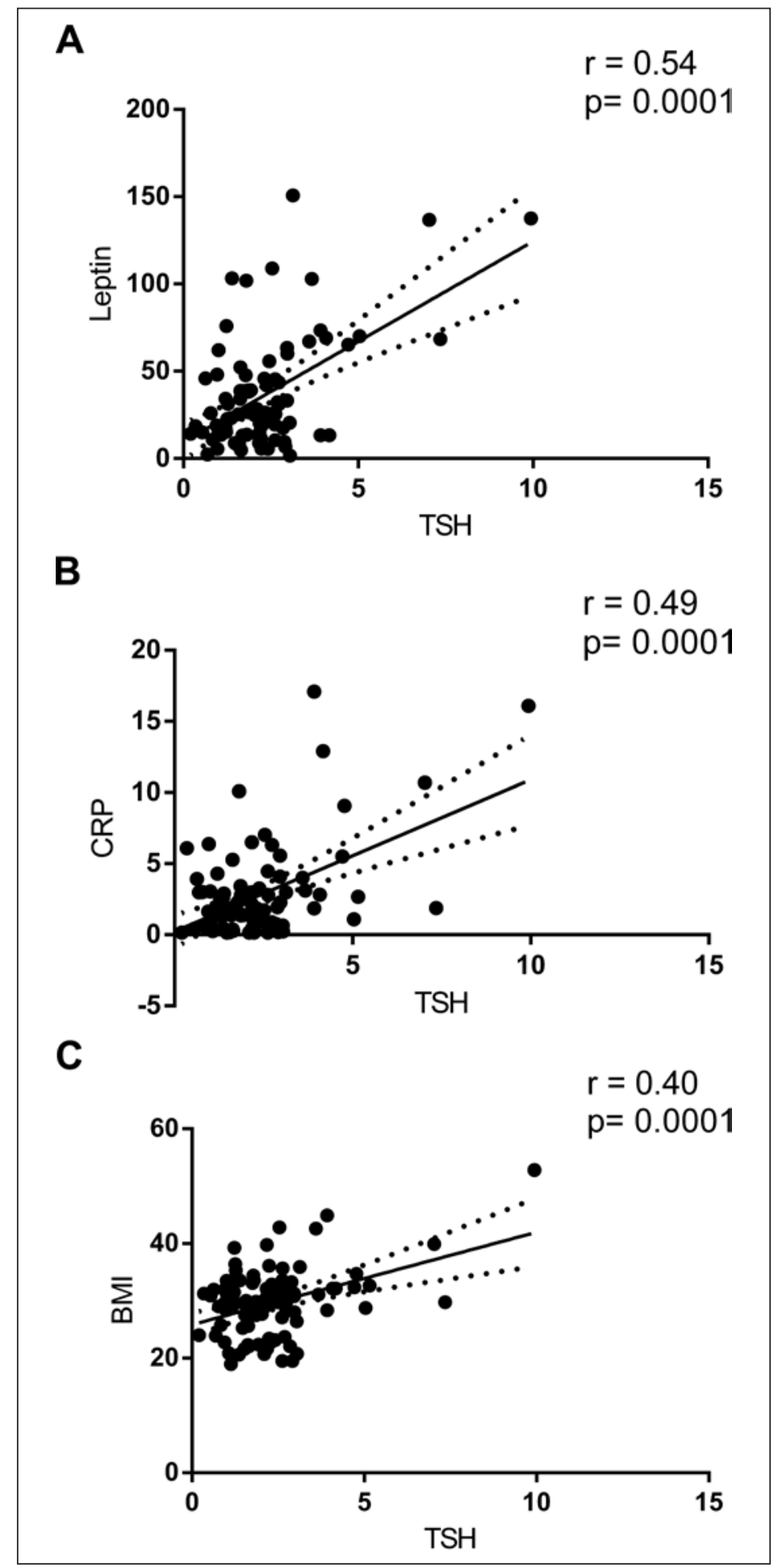

B

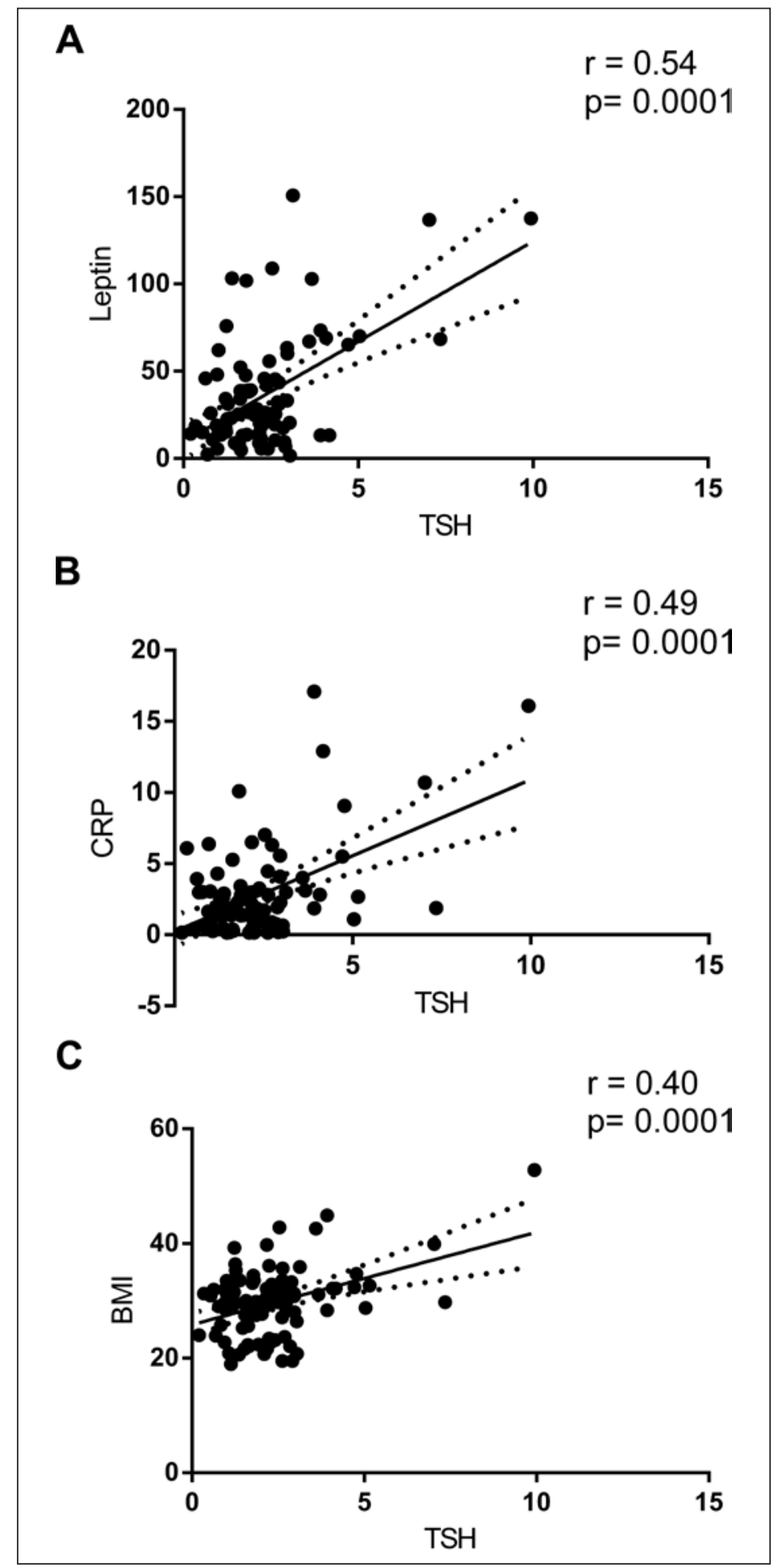

C

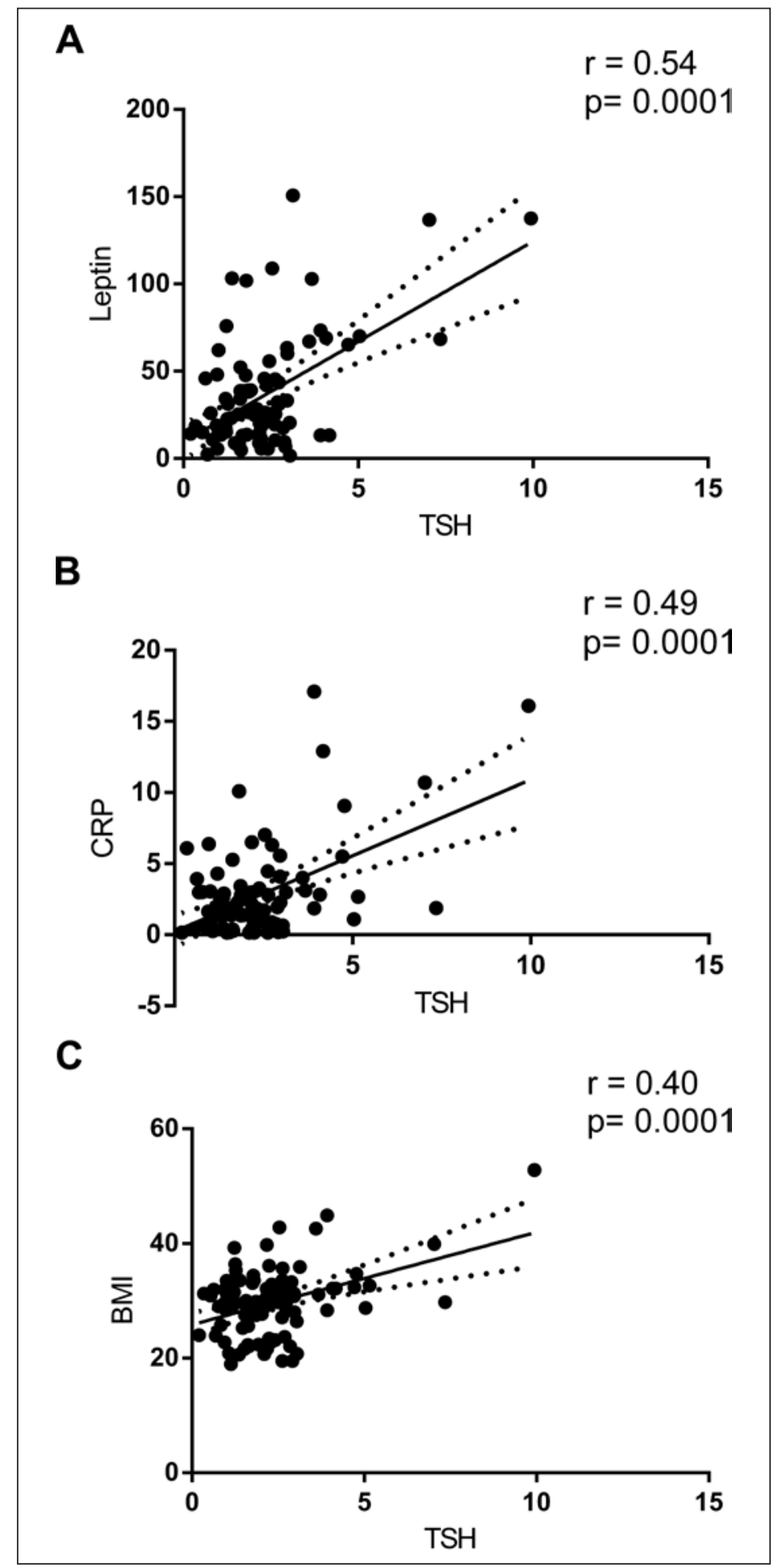

Fig. 1. Pearson correlations of TSH with leptin, CRP, and BMI. Correlations of leptin (A), CRP (B), and BMI (C) with TSH.

had more activation of the HPT axis with higher serum TSH and thyroid hormones. Previously, it has also been postulated that thyroid function abnormalities in obesity may be caused by peripheral thyroid hormone resistance or altered TSH bioactivity, or they may constitute an adaptive process aiming to increase resting energy expenditure, known as adaptive thermogenesis $[20,21]$. In addition, reasons for the elevated TSH levels among diabetics are still 
Yu et al.: Leptin and TSH Alterations after RYGB

Fig. 2. Pearson correlation analysis of $\Delta$ TSH with $\Delta$ leptin.

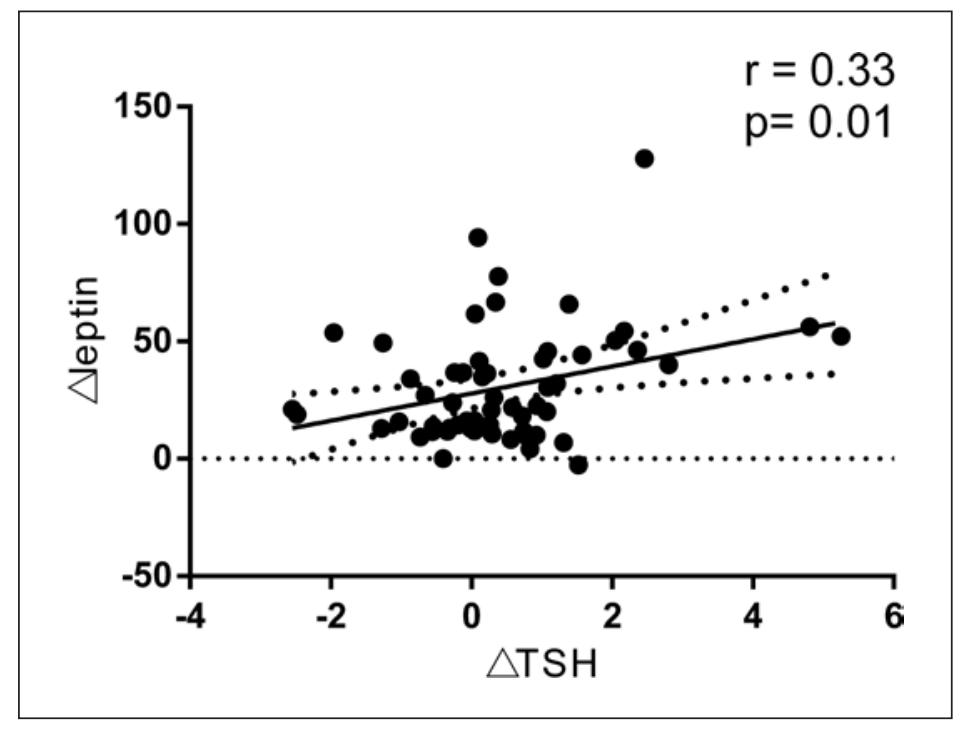

uncertain. Previous studies suggested that leptin levels were higher in diabetics [22], which might stimulate TSH synthesis via the HPT axis. Therefore, a decline in TSH release and $\mathrm{FT}_{3} /$ $\mathrm{FT}_{4}$ concentrations may contribute to the compensatory reductions in energy expenditure and catabolism that typically accompany weight loss and diabetes remission induced by RYGB.

Our observations extend previous results indicating that TSH was significantly positively correlated with BMI, CRP, and leptin. Furthermore, multiple stepwise linear regression indicated that leptin and CRP were independent factors affecting TSH. Nyrnes et al. [23] found normal serum TSH levels were significantly and positively associated with BMI in nonsmokers. Furthermore, during a 7-year follow-up period, they found that an increase in TSH level was associated with an increase in BMI. Bétry et al. [24] have reported that serum TSH was found to be positively correlated with both BMI and leptin in 800 obese subjects. After performing multiple regression analysis, TSH and leptin levels maintained an independent association with BMI. In addition, subjects in the fourth quartile of TSH distribution displayed significantly higher BMI and leptin levels than subjects in the first quartile.

The results on the association between CRP and thyroid function were inconsistent. A cross-sectional study did not find any association between CRP and TSH levels in 1,551 euthyroid subjects and 57 subjects with subclinical hypothyroidism [25]. However, Yu et al. [26] demonstrated that TSH was positively associated with CRP in a cohort of 2,494 Taiwanese adults, which was in line with our results. A possible explanation is that obesity and T2DM are 2 potential mechanisms as well as important contributors that regulate the effect of systemic inflammation on thyroid function.

Although the associations between weight loss and the changes in thyroid hormones have been found in previous studies, the underlying reasons responsible are not well understood. Our results revealed that serum leptin level declined proportionally to the magnitude of TSH, which indicated that leptin might mediate the change in thyroid function after RYGBinduced weight loss. Thyroid hormones and leptin play essential roles in the regulation of body energy balance through similar stimulating effects on energy expenditure. The main action of leptin is to modulate the amount of fat, leading to a decrease in appetite and food intake. It has also been shown to stimulate centrally the transcription of thyrotropin-releasing hormone and TSH in consequence [27]. Ortiga-Carvalho et al. [28] demonstrated that systemically administered leptin at an appropriately low dose had an acute stimulatory effect on TSH 
secretion in fed rats. Moreover, Radwanska et al. [29] found that exogenous leptin at doses of $10^{-10}$ to $10^{-6} \mathrm{~mol} / \mathrm{L}$ elevated TSH secretion from ovine pituitary cells in vitro, and this phenomenon might be mediated by the effect of nitric oxide. Since leptin is an adipocytokine that regulates energy homeostasis and body weight, there could be cross talk between adipose tissue and the HPT axis. A reduction in leptin following weight loss induced by RYGB would lead to decreased TSH secretion.

To our knowledge, our present study is the first to reveal that the normalization of serum TSH after RYGB is associated with decreased leptin in euthyroid patients with obesity and T2DM. One of the strengths is that our study subjects belong to an Asian population with a relatively lower BMI than in the studies previously performed. The second is that the normal control group was set up to determine whether leptin and thyroid hormone levels could return to normal control levels in the RYGB group after weight loss.

In conclusion, although the exact mechanism leading to a decrease in TSH following RYGB remains not well understood, a decrease in leptin after weight loss induced by RYGB may partially mediate this effect.

\section{Acknowledgments}

This study was supported by grants from the National Key Research and Development Project of China (2016YFA0502003), Key Program of the Shanghai Municipality for Basic Research (11JC1409600), Shanghai SJTUSM Biobank, National Natural Science Foundation of China (81670791, 81500625), and the Municipal Natural Science Foundation of Shanghai (17ZR1421200).

\section{Statements of Ethics}

According to the World Medical Association's recommendations, this study was approved by the Sixth People's Hospital Ethics Committee of the Shanghai Jiao Tong University. All participants gave written informed consent before the study. The retrospective study identifier in clinicaltrials.gov is NCT02217943.

\section{Disclosure Statement}

The authors have no conflicts of interest to disclose.

\section{Authors' Contributions}

H.Y., W.J., and Y.B. designed the study. M.Z., F.L., J.P., and J.L. collected and processed the data. M.Z., J.H., Y.T., and P.Z. analyzed the data. H.Y., W.J., Y.B., J.P., and M.Z. participated in data interpretation and manuscript preparation. 
Yu et al.: Leptin and TSH Alterations after RYGB

\section{References}

1 Ng M, Fleming T, Robinson M, Thomson B, Graetz N, Margono C, et al. Global, regional, and national prevalence of overweight and obesity in children and adults during 1980-2013: a systematic analysis for the Global Burden of Disease Study 2013. Lancet. 2014 Aug;384(9945):766-81.

2 Michalaki MA, Vagenakis AG, Leonardou AS, Argentou MN, Habeos IG, Makri MG, et al. Thyroid function in humans with morbid obesity. Thyroid. 2006 Jan;16(1):73-8.

3 Liu G, Liang L, Bray GA, Qi L, Hu FB, Rood J, et al. Thyroid hormones and changes in body weight and metabolic parameters in response to weight loss diets: the POUNDS LOST trial. Int J Obes. 2017 Jun;41(6):878-86.

4 Mwafy S, Yassin M, Mousa R. Thyroid hormones, lipid profile and anthropometric changes after programmed weight loss in Palestinian obese adult females. Diabetes Metab Syndr. 2018 May;12(3):269-73.

5 Yang W, Lu J, Weng J, Jia W, Ji L, Xiao J, et al.; China National Diabetes and Metabolic Disorders Study Group. Prevalence of diabetes among men and women in China. N Engl J Med. 2010 Mar;362(12):1090-101.

6 Gu Y, Li H, Bao X, Zhang Q, Liu L, Meng G, et al. The Relationship Between Thyroid Function and the Prevalence of Type 2 Diabetes Mellitus in Euthyroid Subjects. J Clin Endocrinol Metab. 2017 Feb;102(2):434-42.

7 Schauer PR, Bhatt DL, Kirwan JP, Wolski K, Aminian A, Brethauer SA, et al; STAMPEDE Investigators. Bariatric Surgery versus Intensive Medical Therapy for Diabetes - 5-Year Outcomes. N Engl J Med. 2017 Feb;376(7): 641-51.

8 Yu H, Zhang L, Bao Y, Zhang P, Tu Y, Di J, et al. Metabolic Syndrome After Roux-en-Y Gastric Bypass Surgery in Chinese Obese Patients with Type 2 Diabetes. Obes Surg. 2016 Sep;26(9):2190-7.

9 Fallahi P, Ferrari SM, Camastra S, Politti U, Ruffilli I, Vita R, et al. TSH Normalization in Bariatric Surgery Patients After the Switch from L-Thyroxine in Tablet to an Oral Liquid Formulation. Obes Surg. 2017 Jan; 27(1):78-82.

10 Liu F, Di J, Yu H, Han J, Bao Y, Jia W. Effect of Roux-en-Y gastric bypass on thyroid function in euthyroid patients with obesity and type 2 diabetes. Surg Obes Relat Dis. 2017 Oct;13(10):1701-7.

11 Endo T, Kobayashi T. Expression of functional TSH receptor in white adipose tissues of hyt/hyt mice induces lipolysis in vivo. Am J Physiol Endocrinol Metab. 2012 Jun;302(12):E1569-75.

12 Campfield LA, Smith FJ, Guisez Y, Devos R, Burn P. Recombinant mouse OB protein: evidence for a peripheral signal linking adiposity and central neural networks. Science. 1995 Jul;269(5223):546-9.

13 Santini F, Galli G, Maffei M, Fierabracci P, Pelosini C, Marsili A, et al. Acute exogenous TSH administration stimulates leptin secretion in vivo. Eur J Endocrinol. 2010 Jul;163(1):63-7.

14 Yu H, Di J, Bao Y, Zhang P, Zhang L, Tu Y, et al. Visceral fat area as a new predictor of short-term diabetes remission after Roux-en-Y gastric bypass surgery in Chinese patients with a body mass index less than $35 \mathrm{~kg} /$ m2. Surg Obes Relat Dis. 2015 Jan-Feb;11(1):6-11.

15 Moulin de Moraes CM, Mancini MC, de Melo ME, Figueiredo DA, Villares SM, Rascovski A, et al. Prevalence of subclinical hypothyroidism in a morbidly obese population and improvement after weight loss induced by Roux-en-Y gastric bypass. Obes Surg. 2005 Oct;15(9):1287-91.

16 Chikunguwo S, Brethauer S, Nirujogi V, Pitt T, Udomsawaengsup S, Chand B, et al. Influence of obesity and surgical weight loss on thyroid hormone levels. Surg Obes Relat Dis. 2007 Nov-Dec;3(6):631-5; discussion 635-6.

17 MacCuish A, Razvi S, Syed AA. Effect of weight loss after gastric bypass surgery on thyroid function in euthyroid people with morbid obesity. Clin Obes. 2012 Feb;2(1-2):25-8.

18 Vettor R, Mingrone G, Manco M, Granzotto M, Milan G, Scarda A, et al. Reduced expression of uncoupling proteins- 2 and -3 in adipose tissue in post-obese patients submitted to biliopancreatic diversion. Eur J Endocrinol. 2003 May;148(5):543-50.

19 Guan B, Chen Y, Yang J, Yang W, Wang C. Effect of Bariatric Surgery on Thyroid Function in Obese Patients: a Systematic Review and Meta-Analysis. Obes Surg. 2017 Dec;27(12):3292-305.

20 Emerson CH. Anthropomorphic thyroidopathies? Thyroid. 2010 Nov;20(11):1195-7.

21 Reinehr T. Obesity and thyroid function. Mol Cell Endocrinol. 2010 Mar;316(2):165-71.

22 Al-Hamodi Z, Al-Habori M, Al-Meeri A, Saif-Ali R. Association of adipokines, leptin/adiponectin ratio and C-reactive protein with obesity and type 2 diabetes mellitus. Diabetol Metab Syndr. 2014 Sep;6(1):99.

23 Nyrnes A, Jorde R, Sundsfjord J. Serum TSH is positively associated with BMI. Int J Obes. 2006 Jan;30(1):100-5.

24 Bétry C, Challan-Belval MA, Bernard A, Charrié A, Drai J, Laville M, et al. Increased TSH in obesity: evidence for a BMI-independent association with leptin. Diabetes Metab. 2015 Jun;41(3):248-51.

25 Hueston WJ, King DE, Geesey ME. Serum biomarkers for cardiovascular inflammation in subclinical hypothyroidism. Clin Endocrinol (Oxf). 2005 Nov;63(5):582-7.

26 Yu YT, Ho CT, Hsu HS, Li CI, Davidson LE, Liu CS, et al. Subclinical hypothyroidism is associated with elevated high-sensitive C-reactive protein among adult Taiwanese. Endocrine. 2013 Dec;44(3):716-22.

27 Sanyal D, Raychaudhuri M. Hypothyroidism and obesity: an intriguing link. Indian J Endocrinol Metab. 2016 Jul-Aug;20(4):554-7.

28 Ortiga-Carvalho TM, Oliveira KJ, Soares BA, Pazos-Moura CC. The role of leptin in the regulation of TSH secretion in the fed state: in vivo and in vitro studies. J Endocrinol. 2002 Jul;174(1):121-5.

29 Radwanska P, Kosior-Korzecka U. Effect of leptin on thyroid-stimulating hormone secretion and nitric oxide release from pituitary cells of ewe lambs in vitro. J Physiol Pharmacol. 2014 Feb;65(1):145-51. 